\title{
Immunohistochemistry: A Valuable Tool in the Management of Common Female Genital Tract Tumors_-A Clinicians' Perspective
}

\author{
Rajesh Panicker ${ }^{1}$, Shyamoli Mustafa ${ }^{2}$
}

\begin{abstract}
Immunohistochemistry (IHC) is a laboratory technique that can be used by the pathologist to add value to the diagnosis. The basic principle of $\mathrm{IHC}$ involves using specific antibodies to go and get attached to specific antigens present on biological tissue and then visualizing the antigenantibody reaction thus identifying the cell type using its antigenic characteristics.

The use of IHC in histopathological diagnosis can ensure a precise diagnosis thus helping the clinician to make appropriate therapeutic choices related to the management of common genital tract tumors. Cervix: IHC can help in differentiating reactive changes from cervical intraepithelial lesions. IHC can also improve the specificity of Pap smears while screening for squamous intraepithelial lesions. Corpus uteri: IHC has been used to identify cases of primary adenocarcinoma of the uterus metastasizing to the cervix and also to differentiate between type I and type II endometrial carcinoma. IHC can also be utilized to improve the positive predictive value of endometrial sampling to preoperatively diagnose uterine leiomyosarcomas. Ovary: One major problem faced by the clinician is the early intraoperative diagnosis of ovarian malignancy and IHC helps in obtaining an early and accurate diagnosis. Gestational trophoblastic diseases: The use of IHC techniques can help in accurate diagnosis of complete hydatidiform mole. Vulva: Coexistence of high-grade vulvar intraepithelial neoplasia or verrucous carcinoma of the vulva with condyloma acuminata, which is a benign condition, can be picked up using IHC and this is a big advantage to the clinician to plan a treatment protocol.

Though IHC is an important tool, there are issues of standardization and reproducibility that need to be addressed. IHC is yet to evolve into a standalone method of laboratory diagnosis and must be used in conjunction with clinical assessment and histopathological findings.

Keywords: Accurate, Antigen-antibody, Cervix, Immunomarkers, Ovary, Standardization, Uterus, Vulva.

Journal of South Asian Federation of Obstetrics and Gynaecology (2021): 10.5005/jp-journals-10006-1880
\end{abstract}

\section{INTRODUCTION}

In the past, the pathologist used to make a diagnosis of neoplasia solely by examining the morphological characteristics of tissue using paraffin sections stained with hematoxylin and eosin. Occasionally, they used special stains to establish a diagnosis. However, for present-day practice, the clinician requires a precise and accurate diagnosis and the pathologist needs to incorporate additional tools in making the pathological diagnosis.

Immunohistochemistry (IHC) is one such tools used by the pathologist to add value to the diagnosis by improving accuracy and hence prognosis. Today, IHC is useful to study disease pathogenesis and to prepare tailor-made therapeutic decisions for individual patients.

$\mathrm{IHC}$ is a laboratory technique that can help to confirm primary tumor cell type and can be of use in identifying the possible origin of metastatic cancer from an unknown primary site. It can also help to detect early local invasion and lymph node metastasis by exploiting the principle of identifying typical cellular components by utilizing their antigenic characteristics. ${ }^{2}$ The basic principle of IHC involves using specific antibodies to go and get attached to specific antigens present on biological tissue and then visualizing the antigen-antibody reaction thus identifying the cell type using its antigenic characteristics. ${ }^{1}$ Taylor and Burns in 1974, from Oxford University, were the first to successfully demonstrate the antigenic properties in cells from routinely processed, formalinfixed, paraffin-embedded tissues.
${ }^{1}$ Department of Obstetrics and Gynecology, AIMST University, Bedong, Malaysia

${ }^{2}$ Department of Pathology, Faculty of Medicine, Quest International University, Ipoh, Perak, Malaysia

Corresponding Author: Rajesh Panicker, Department of Obstetrics and Gynecology, AIMST University, Bedong, Malaysia, Phone: +91 601126146711, e-mail: rpanicker@rediffmail.com

How to cite this article: Panicker R, Mustafa S. Immunohistochemistry: A Valuable Tool in the Management of Common Female Genital Tract Tumors-A Clinicians' Perspective. J South Asian Feder Obst Gynae 2021;13(2):131-134.

Source of support: Nil

Conflict of interest: None

\section{Principle of Immunohistochemistry}

As mentioned before, IHC is a laboratory technique for the identification of cellular characteristics even though the morphological characteristics are unclear. It employs an immunomarker which is an antibody to the antigen on the cell, resulting in an antigen-antibody reaction. This is subsequently visualized using a chromogenic detector which is an enzyme, conjugated or labeled to the antibody, which subsequently cleaves a substrate to produce a colored precipitate. This process is called immunostaining and this can be performed by two methods: a direct method or an indirect method. The indirect method is more

(0) Jaypee Brothers Medical Publishers. 2021 Open Access This article is distributed under the terms of the Creative Commons Attribution 4.0 International License (https://creativecommons.org/licenses/by-nc/4.0/), which permits unrestricted use, distribution, and non-commercial reproduction in any medium, provided you give appropriate credit to the original author(s) and the source, provide a link to the Creative Commons license, and indicate if changes were made. The Creative Commons Public Domain Dedication waiver (http://creativecommons.org/publicdomain/zero/1.0/) applies to the data made available in this article, unless otherwise stated. 
sensitive and also more convenient and hence more popular. In this method, unlabeled antibodies are used in the first step to get attached to the antigen. Then the second layer of labeled antibodies against the primary antibody is added and this is then visualized. The avidin-biotin complex is most commonly used in indirect immunohistochemical methods, where the primary antibody is attached to biotin and the secondary antibody is an avidin-biotin complex that is linked to an enzyme. ${ }^{2}$ The enzyme most widely used is horseradish peroxidase, whereas 3,3'-diaminobenzidine tetrachloride is the most favored chromogen used in IHC. With the advances in immunopathology, newer diagnostic and prognostic IHC markers are being identified, and as a result, the scope and output of $\mathrm{IHC}$ in the laboratory are rising.

Having elaborated on the importance of IHC, we must also caution that there are numerous pitfalls in $\mathrm{IHC}^{3}$ At times, unexpected staining reactions could occur and this could result in potential diagnostic problems. So it stands to reason that the interpretation of $\mathrm{IHC}$ requires overall correlation with the clinical, radiological, gross pathological, and microscopic findings. ${ }^{4}$

\section{IHC in Aid of the Clinician for the Better Management of Common Female Genital Tract TUMORS}

\section{Uterine Cervix}

$\mathrm{IHC}$ is a valuable adjunct while performing cervical histopathology. Diagnosing squamous intraepithelial lesions from a cervical biopsy, especially in patients with normal colposcopic findings, is fraught with high false positivity as reactive changes could also mimic the morphological changes of squamous intraepithelial lesions. This is important from the viewpoint of the clinician because reactive changes do not require any treatment. Reactive changes include reparative changes, cautery artifact, menopausal changes, and immature squamous metaplasia. IHC using immunomarkers for p16 antigen (tumor suppressor gene), which is most widely used as a surrogate marker for human papillpoma virus (HPV) infection, is decisive in distinguishing reactive changes from true squamous intraepithelial lesions. ${ }^{5}$

IHC also comes in handy while differentiating cervical glandular dysplasia and adenocarcinoma in situ, from reactive changes. Of course, here the markers used are different and would include CD10 and HK 1083. ${ }^{3}$

Can we combine immunocytochemistry with the Pap smear and improve the sensitivity and specificity of the screening for preinvasive lesions of the cervix? This is a question researchers are working on and today the combination of liquid-based Pap test with immunocytochemistry for HPV DNA has improved the sensitivity of the Pap test. The problem, however, with this combination is that though the sensitivity of the screening process does increase, the specificity does not improve significantly. ${ }^{6}$ These combination kits have not yet been approved by the USFDA.

To categorize a poorly differentiated cervical cancer as squamous cell carcinoma or adenocarcinoma can often be difficult, and this is a dilemma for the clinician as the management of the two conditions is different. Markers such as CK5/6, p63, cam5.2, CK7, and CK20 are useful in differentiating between the two. CK5/6 and p63 stain the squamous cell carcinoma whereas adenocarcinoma will be positive for cam5.2 and CK7, but negative for CK20. ${ }^{7}$

IHC can also be used to specifically diagnose neuroendocrine carcinoma of the cervix which is a rare variant with a poor prognosis.
The most commonly used immunomarker for neuroendocrine carcinoma of the cervix is synaptophysin. ${ }^{8}$

\section{Corpus Uteri}

The histopathological differentiation between a primary cervical adenocarcinoma and endometrial adenocarcinoma with cervical invasion becomes difficult, because very often, the tissue sample provided for biopsy is very small and imprecise. This could be a dilemma for the clinician since the management of the two conditions is different. McCluggage GW and Jenkins D effectively showed that IHC (usingP16, vimentin, ER, PR, CEA, ProExC) is an effective method to differentiate these two conditions whenever there is a doubt. ${ }^{9}$

Recently, the subdivision of endometrial carcinoma into type I (endometrioid) and type II (serous papillary), based on histology, has altered perspectives of management. This is because type II tumors have a worse prognosis and as a result, management protocols for type II tumors tend to be more aggressive. IHC for confirmation of the typing of endometrial carcinoma, can be done using p53 immunoreactivity, which is seen in more than $80 \%$ of type II tumors as opposed to only $20 \%$ of type I tumors. Other immunomarkers such as MIB 1, p16, and estrogen and progesterone receptors (ER\&PR) can also be used to differentiate type I and II tumors. ${ }^{10}$

Another problem faced by the pathologist is assigning the appropriate FIGO staging of endometrial carcinoma based on the depth of myometrial invasion. This is all the more so in cases of concomitant adenomyosis, since the depth of myometrial invasion needs differentiation from the focus of adenomyosis. The immunomarker CD10 is useful in making this distinction and can help in precisely assessing myometrial invasion. ${ }^{11}$

One of the most difficult differential diagnoses in clinical gynecology concerns the preoperative distinction between leiomyomas (uterine fibroid) and leiomyosarcomas. Preoperative identification of uterine sarcomas will allow for better preoperative evaluation and appropriate surgical planning. Given the aggressive nature of sarcomas, a careful preoperative confirmation of a leiomyosarcoma will ensure better evaluation for systemic metastases and can result in better treatment outcomes. Nisha Bansal et al. studied the utility of preoperative endometrial sampling for the detection of uterine sarcomas and they found that histopathology has a low predictive value in diagnosing uterine sarcomas. ${ }^{12}$ However using IHC and a panel of immunomarkers MIB 1, p53, ER, PR, and $\mathrm{BCl}-2$, it was possible to improve the positive predictive value of endometrial sampling to preoperatively diagnose uterine leiomyosarcomas. ${ }^{12,13}$

\section{Ovary}

One major problem faced by the clinician is the intraoperative diagnosis of ovarian malignancy. Presently, the system employed uses frozen section histopathology to identify malignant cells so that the appropriate staging procedure could be performed by the surgeon. But as we know, the specificity of frozen section histopathology is poor. Rapid immunostaining of frozen sections within a short time span would be very helpful for intraoperative diagnosis. Research is ongoing to develop protocols for quick diagnosis using the enhanced polymer one step (EPOS) IHC technique and this could be tried out for ovarian malignancies. This procedure takes only 12 minutes, thus enabling rapid immunostaining on intraoperative frozen sections. Another technique being tried is the incorporation of immunoperoxidase staining techniques, right at the beginning while processing the frozen tissue. This process is even faster than EPOS and trials are on while performing Mohs micrographic surgery, 
for dermatological cancers. There is scope for utilizing this technique for epithelial ovarian tumors, the main advantage being, rapid intraoperative tumor classification. ${ }^{14}$

IHC is also helpful in differentiating between borderline ovarian tumors (BOTs) from invasive epithelial ovarian cancers. This is significant for the clinician as management of BOT is more conservative and fertility-sparing surgery could be offered to younger patients by the clinician. The immunomarkers used for this differentiation include P53, Ki67, and MM1. ${ }^{15} \mathrm{IHC}$ can also be used to differentiate between serous BOT and mucinous BOT. This is of importance to the clinician since mucinous BOT has a worse prognosis and requires more aggressive treatment.

Another application of $\mathrm{IHC}$ is in differentiating primary ovarian malignancy from secondary ovarian involvement, especially from the liver and gastrointestinal primaries. The panel of immunomarkers for this differentiation could include CEA, low molecular weight cytokeratins (CK7, CK8), and vimentin. ${ }^{16}$

\section{Gestational Trophoblastic Disease}

Gestational trophoblastic disease (GTD) includes diseases ranging from the common hydatidiform mole to the rarer placental site trophoblastic tumors and choriocarcinoma. In clinical practice, though there are many clinical pointers, the final diagnosis remains histopathological. However, the histological diagnosis of GTD continues to have significant diagnostic inaccuracy with marked interobserver and intraobserver variabilities, even among expert pathologists. ${ }^{17}$ The clinical, ultrasonographic, and histopathological features of complete hydatidiform mole often mimic those of partial hydatidiform mole and missed miscarriage with a chromosomal abnormality, especially in very early pregnancy. This fact puts the clinician in a quandary since the management and follow-up of these conditions are very different. The immunomarker p57 is a very useful marker in confirming the diagnosis of the complete hydatidiform mole. ${ }^{17}$ The differentiation between partial hydatidiform mole and missed miscarriage with chromosomal abnormality is more difficult. In such cases, DNA extraction with PCR may help in the diagnosis. ${ }^{17}$

\section{Vulva}

Condyloma acuminata or venereal warts are exclusively caused by HPV subtypes $(6,11,42,43,44)$ which are known to have minimal malignant potential. Since premalignant or invasive lesions such as high-grade vulvar intraepithelial neoplasia of warty type or verrucous carcinoma of the vulva may often appear similar to or coexist within venereal warts, histopathological confirmation is mandatory. Histopathological diagnosis of vulvar condyloma acuminata depends upon nonspecific architectural characteristics. Hence accurate confirmation can be made using the immunohistochemical marker MIB1. The presence of coexisting malignant or premalignant lesions needs confirmation using immunomarkers p16 and p53, which are specific for HPV-induced vulvar carcinoma. These markers can also be used to differentiate between vulvar carcinoma caused by oncogenic HPV and those not induced by oncogenic HPV. This differentiation is important to the clinician since prognosis and treatment protocols are different.

\section{Conclusion}

$\mathrm{IHC}$ has the potential to transform surgical pathology into a more objective science. The increasing use of IHC and the demands for comparison of qualitative and semiquantitative findings among an increasing number of laboratories have resulted in a growing focus on reproducibility and a new emphasis on standardization. Despite the widespread use of IHC as a biomarker validation tool, there are no universally accepted guidelines for the use of particular antibodies for specific pathologies. ${ }^{18}$ As we go into the future this needs attention.

Clinicians are now tending to look at genital tract tumors, through the prism of IHC. This is because it has improved accuracy in diagnosis and reduced ambiguity in management. However, though IHC is a great tool for the clinician, for the histopathologist, traditional morphologic assessment should still remain the first step in making a diagnosis.

\section{References}

1. Ramos-Vara JA, Miller MA. When tissue antigens and antibodies get along: revisiting the technical aspects of immunohistochemistry the red brown and black technique. Vet Path 2014;51(I):42-87. DOI: 10.1177/0300985813505879.

2. Idikio HA. Immunohistochemistry in diagnostic surgical pathology: contributions of protein life-cycle, use of evidence-based methods and data normalization on interpretation of immunohistochemical stains. Int J Clin Exp Pathol 2009;3(2):169-176. PMID: 20126585 PMCID: PMC2809997. https://ncbi.nlm.nih.gov/pmc/articles/PMC2809997.

3. Yaziji H, Barry T. Diagnostic immunohistochemistry: what can go wrong? Adv Anat Pathol 2006;13(5):238-246. DOI: 10.1097/01. pap.0000213041.39070.2f.

4. Glenn McCluggage W. Immunohistochemistry as a diagnostic aid in cervical pathology. Pathology 2007;39(1):97-111. DOI: 10.1080/00313020601123961.

5. Kaspar HG, Crum CP. The utility of immunohistochemistry in the differential diagnosis of gynecologic disorders. Arch Pathol Lab Med 2015;139(1):39-54. DOI: 10.5858/arpa.2014-0057-RA.

6. Morgan TK, Berlin M. Immunocytochemical analysis of the cervical pap smear. In: Keppler D, Lin A, editors. Methods in molecular biology, vol. 1249. New York, NY: Humana Press; 2015. DOI: 10.1007/978-1-49392013-6_15.

7. Selves J, Long-Mira E, Mathieu MC, et al. Immunohistochemistry for diagnosis of metastatic carcinomas of unknown primary site. Cancers (Basel) 2018;10(4):108. DOI: 10.3390/cancers10040108.

8. Rekhi B, Patil B, Deodhar KK, et.al. Spectrum of neuroendocrine carcinomas of the uterine cervix, including histopathologic features, terminology, immunohistochemical profile, and clinical outcomes in a series of 50 cases from a single institution in India. Ann Diagn Pathol 2013;17(1):1-9. DOI: 10.1016/j.anndiagpath.2012.01.009.

9. McCluggage GW, Jenkins D. p16 immunoreactivity may assist in the distinction between endometrial and endocervical adenocarcinoma. Int J Gynecol Pathol 2003;22(3):231-235. DOI: 10.1097/01.PGP.0000055172.04957.2F.

10. Mittal K, Soslow R, McCluggage WG. Application of immunohistochemistry to gynecologic pathology. Arch Pathol Lab Med 2008;132(3):402-423. DOI: 10.1043/1543-2165(2008) 132[402:AOITGP]2.0.CO;2.

11. Nascimento AF, Hirsch MS, Cviko A, et.al. The role of CD10 staining in distinguishing invasive endometrial adenocarcinoma from adenocarcinoma involving adenomyosis. Mod Pathol 2003;16(1):2227. DOI: 10.1097/01.MP.0000043523.03519.FC.

12. Bansal N, Herzog TJ, Burke W, et al. The utility of preoperative endometrial sampling for the detection of uterine sarcomas. Gynecol Oncol 2008;110(1):43-48. DOI: 10.1016/j.ygyno.2008.02.026.

13. Rubisz P, Ciebiera M, Hirnle L, et al. The usefulness of immunohistochemistry in the differential diagnosis of lesions originating from the myometrium. Int J Mol Sci 2019;20(5):1136. DOI: 10.3390/ijms20051136.

14. El Tal AK, Abrou AE, Stiff MA, et al. Immunostaining in Mohs micrographic surgery: a review. Dermatol Surg 2010;36(3):275-290. DOI: 10.1111/j.1524-4725.2009.01432.x. 
15. Giurgea LN, Ungureanu C, Mihailovici MS. The immunohistochemical expression of p53 and Ki67 in ovarian epithelial borderline tumors. Correlation with clinicopathological factors. Rom J Morphol Embryol 2012;53(4):967-973. PMID: 23303020. http://doi.org/10.47162/RJME.

16. Geller SA, Dhall D, Alsabeh R. Application of immunohistochemistry to liver and gastrointestinal neoplasms: liver, stomach, colon, and pancreas. Arch Pathol Lab Med 2008;132(3):490-499. DOI: 10.1043/1543-2165(2008)132[490:AOITLA]2.0.CO;2.
17. Buza N, Hui P. Immunohistochemistry and other ancillary techniques in the diagnosis of gestational trophoblastic diseases. Semin Diagn Pathol 2014;31(3):223-232. DOI: 10.1053/ j.semdp.2014.03.004.

18. O'Hurley G, Sjöstedt E, Rahman A, et.al. Garbage in, garbage out: a critical evaluation of strategies used for validation of immunohistochemical biomarkers. Mol Oncol 2014;8(4):783-798. DOI: 10.1016/j.molonc.2014.03.008. 